\title{
Research on semi-active vibration isolation system based on electromagnetic spring
}

\author{
Jiawei Xu* ${ }^{*}$ Xuefeng Yang, Wei Li, Jiayu Zheng, Yuqiao Wang, and Mengbao Fan \\ School of Mechatronic Engineering, China University of Mining and Technology, Xuzhou Jiangsu 221116, PR China
}

Received: 29 October 2018 / Accepted: 20 September 2019

\begin{abstract}
This paper proposes a semi-active variable stiffness vibration isolation system based on electromagnetic spring for the low-frequency vibration isolation of mass-varying objects. It is achieved by four straight leaf springs in parallel to an electromagnetic spring system composed of a single electromagnet and a permanent magnet. The equivalent magnetic circuit method is used to compute electromagnetic force of the electromagnetic spring system, and mathematical model of the semi-active vibration isolation system is established according to Maxwell's equations. The nonlinear mathematical model is linearized at the equilibrium point by using the Taylor series expansion theorem to establish linear state-space representation of the system, and then using the traditional PID control method, a double closed-loop feedback control system of the inner current loop and outer location loop is designed. By controlling the current in the coil, the equivalent stiffness and electromagnetic force of the system are variable to achieve semi-active control. Furthermore, the control block diagram of the semi-active vibration isolation system is built based on Simulink software, then make a simulation analysis to the vibration isolation performance of the system and compare the effects of vibration isolation with inner current loop control and without inner current loop control, respectively. Finally, the experiments prove the correctness of the theory. It concludes that this semi-active vibration isolation system is a vibration isolation system with broad application prospects, which has fast current response, high vibration isolation efficiency, and an excellent vibration isolation effect for the low-frequency disturbance of mass-varying objects.
\end{abstract}

Keywords: Low frequency vibration isolation / variable stiffness / semi-active control / electromagnetic spring / equivalent magnetic circuit

\section{Introduction}

Vibration isolation technology is the most widely applied in the field of vibration control, and it has a wide range of applications in the field of civil engineering, aerospace, automobile, ships and precision manufacturing and processing, etc. Vibration isolation is generally categorized as passive, active and semi-active vibration isolation [1-4]. Passive vibration isolation is widely employed to structural vibration isolation because of its simple structure, low power consumption, high stability, etc. However, once the structure of the passive vibration isolation system is determined, the stiffness and transmissibility of the system are determined. Therefore, it can only attenuate the fixed narrow band vibration. Hence, it appears that the traditional vibration isolation system is

\footnotetext{
*e-mail: 412666548@qq.com
}

difficult to obtain the ideal performance for the vibration isolation object with variable mass and it is difficult to efficiently isolate low and ultra-low frequency vibration. However, active vibration isolation system $[5,6]$ can automatically track changes of external disturbance frequency and effectively control the vibration of low frequency and broadband, which can make up for the shortcomings of passive vibration isolation technology. However, for the complex systems, the control systems are also relatively complex, which inevitably increases the energy consumption, descends the control precision and makes the vibration isolation system not work as expected.

Recently, the semi-active vibration isolation, as a compromise between passive and active vibration isolation, has become a research hotspot for researchers both at domestic and foreign $[7,8]$. In comparison to passive vibration isolation system, semi-active vibration isolation system has better vibration isolation performance especially in low frequency range. Compared with active 
vibration isolation system, semi-active vibration isolation system does not require a lot of energy and weight $[9,10]$. In addition, it has a relatively simple control system, low cost and reliable operation. Karnopp [11] first proposed a method of semi-active control, which actively adjusted the traditional passive isolator parameters (stiffness or damping). After that, Guglielmino and Edgo [12] proposed a hydraulically driven friction damper, which was an isolator that modified friction damping of system. But it was rarely used in practice at present on account of the wear and noise caused by friction. In order to avoid friction problems, Suda et al. [13] created a high-efficient electromagnetic damper, original and promising, which adjusted magnitude of damping force by controlling the electromagnetic force generated by loopinduced current. Regarding the variable stiffness, Liu et al. [14] fabricated a vibration isolation system of variable stiffness and damping by using two controllable magnetorheological dampers, which simplified the control system of variable stiffness. Meanwhile, replacing the traditional spring with a new type of spring is also an effective method to improve the vibration isolation performance. In recent years, Wu et al. [15] put forward a semi-active variable stiffness vibration system that replaced the coil spring with the planar spring. It made the device structure compact and effectively eliminated the interference with large frequency variations. Then Wang et al. [16] proposed a magnetorheological elastomer variable stiffness vibration isolation system, using a new type of smart material to change the stiffness of the system to meet the vibration isolation requirements under different working conditions. Subsequently, Wickramasinghe et al. [17] referred to the concept of smart springs and proposed a vibration isolation device based on piezoelectric ceramic actuator to actively change stiffness, damping, or mass, which achieved the semi-active control of vibration.

In addition, Zhu and Yuan et al. [18] presented a typical electromagnetic spring model, which controlled the movement of objects by controlling the current in the electromagnetic coil and generating electromagnetic force to ensure that objects are at a stable and predetermined position. Zhang [19] also utilized the characteristics of the electromagnetic spring to design an electromagnetic active vibration absorber and the control system, and then studied the feature of the absorber in detail. After that, Fung et al. [20] invented an electromagnetic actuator to control vibration. Two permanent magnets are used in the actuator, and one of them is entwined with the coil. Then control the current in the coil by PID control, secondary feedback control and optimal feedback control law. Although vibration is suppressed effectively, the control system is relatively complicated. Zhou [21] developed a variable stiffness vibration isolator with an electromagnetic spring composed of the stator coil and the rotor core, and the adjustability of the stiffness was researched and tested. Pranoto et al. [22] designed a vibration suppression system based on the magnetorheological damper and the electromagnetic spring combined with an electromagnet and a permanent magnet, which used a method of interference cancellation to control the current in the coil to isolate the vibration. Subsequently, $\mathrm{Wu}$ et al. [23] proposed a new hybrid magnetic levitation active vibration isolation system, used PID active closed-loop control and the influence of various control parameters on the vibration isolation performance and control performance of the system is analyzed. The results showed that the hybrid magnetic levitation active vibration isolation system has excellent vibration isolation performance in different frequency bands. Nevertheless, only closed-loop control of position feedback used in the system led to slow current response and a large delay of the system response.

As the above research shows, the semi-active control vibration isolation is generally used to low frequency vibration isolation of mass-varying objects. The semiactive control vibration isolation system relatively decreases the energy consumption and simplifies control system. However, it increases the complexity of the vibration isolation system structure. Hence, in order to avoid the complexity of the structure, a new-style electromagnetic spring is proposed, which has a good characteristic of current control, simple and practical structure. It is an effective method in a semi-active control vibration isolation system. However, due to the non-linear electromagnetic force, there are still two problems. One of them is the difficulty to design the control system, and the other is the large delay of vibration isolation system response caused by the lag of current response, which affect the vibration isolation effect together. In this paper, a semiactive variable stiffness vibration isolation system is designed by using straight leaf spring in parallel to an electromagnetic spring with a hybrid structure composed of a single electromagnet and a permanent magnet, which has a simple structure and is easy to miniaturize. Moreover, the inner current loop is added to the control system to form a double closed-loop control method, which improves the speed of current response and the stability of system response. Simultaneously, the delay of system response is also improved.

This paper focuses on the design and the simulation analysis of the semi-active vibration isolation system based on electromagnetic spring. The structure is as follows: in Section 2, a three-dimensional model of semiactive vibration isolation system and its components are shown. In Section 3, the equivalent magnetic circuit method is used to calculate the electromagnetic repulsion force [24] of electromagnetic spring. Then the mathematical model of semi-active vibration isolation system is built and linearized at the equilibrium point. In Section 4, the linear state-space representation is established according to the linearized mathematical model mentioned above, the control system is designed, and the control block diagram of semi-active vibration isolation system is constructed by means of double closed-loop control method of inner current loop and outer position loop. In Section 5, the dynamic and stiffness of electromagnetic spring is analyzed. In Section 6, based on the Simulink software, the simulation analysis is made regarding the vibration isolation performance of system, which shows the effectiveness of the design. In Section 7 , an experimental investigation is performed on the 

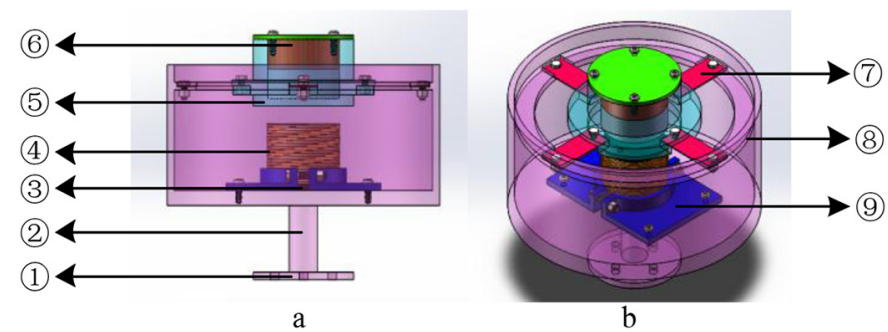

Fig. 1. Three-dimensional figure of semi-active vibration isolation system.

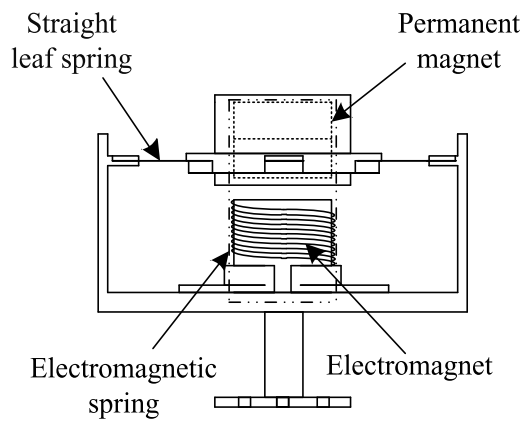

Fig. 2. Diagram of semi-active vibration isolation system structure.

vibration isolation system. In Section 8, the conclusion and outlook for the vibration isolation system is indicated.

\section{Vibration isolator 3D modeling}

As shown in Figure 1, the semi-active vibration isolation system comprises: (1) base, (2) support pillar, (3) iron core, (4) coil, (5) stage, (6) permanent magnet, (7) straight leaf springs, (8) case, and (9) fixture. The coil is wound around the iron core to form an electromagnet that is fixed at the central position of the case by the fixture. The permanent magnet is placed coaxially with the electromagnet in the stage, and there is a certain gap. The four straight leaf springs are placed at equal intervals around the stage, and the two ends bolted to the case and stage, respectively.

The cylindrical permanent magnet and electromagnet are arranged in repulsive configuration, and their magnetic fields are evenly distributed along the central axis. The electromagnet is fixed at the bottom of the case, the permanent magnet is limited to five degrees of freedom by the straight leaf spring and can only be moved up and down vertically. The structure of vibration isolation system is show in Figure 2. When the straight leaf spring with no deformation, it is regarded as the quasi-equilibrium position of the system. Under the external disturbance vibration, the current in the coil is controlled to change the magnitude of electromagnetic force between the electromagnetic springs so that the system could be kept near the quasi-equilibrium position to achieve the purpose of suppressing vibration.

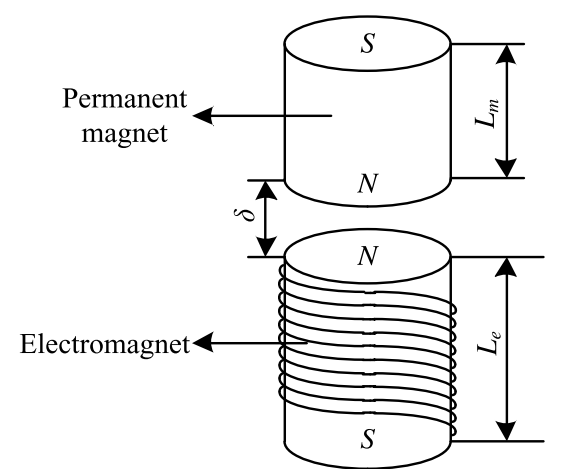

Fig. 3. Schematic diagram of the simplified model of electromagnetic spring system.

\section{Modeling of electromagnetic spring system}

\subsection{Non-linear mathematical model}

The cylindrical permanent magnet and electromagnet are arranged in repulsive configuration and is employed to produce repulsive force, which is used to raise objects. The repulsive force changes with the variation of the gap and current, and the stiffness of the vibration isolation system can be also changed to achieve the purpose of semi-active control. The physical simplified model of the electromagnetic spring system is shown in Figure 3.

The electromagnetic permanent magnetic hybrid structure in this paper adopts the DC power supply, so there is no eddy current loss, and the core loss is small. As shown in Figure 3, the number of turns of the electromagnet coil is $N$, effective length is $L_{e}$, effective cross-sectional area is $S_{e}$ and the current in the coil is $I$. The effective length of the permanent magnet is $L_{m}$, the effective cross-sectional area is $S_{m}$, coercive force is $H_{c}$, magnetic induction strength is $B_{r}$, the size of air gap is $\delta$ and the air magnetic permeability is $\mu_{0}$.

For the calculation of the repulsive force of electromagnetic permanent magnet hybrid structure, this paper uses the equivalent magnetic circuit method. Assumptions: (1) ignore magnetic flux leakage from coils and permanent magnets, (2) magnetic potential is evenly distributed over the air gap and the permanent magnet, i.e. ignore the magnetic resistance of the core, (3) the heat dissipation of the coil is good.

The equivalent magnetic circuit of the system is shown in Figure 4.

As illustrated in the equivalent magnetic circuit model, $U_{m}$ and $R_{m}$ are the magneto motive force and the internal magnetoresistance of the permanent magnet, $U_{e}$ is the magneto motive force generated by the electromagnetic coil, $R_{\delta}$ is the air gap magnetoresistance. According to the basic laws of magnetic circuits:

$$
\begin{gathered}
U_{e}=I N, \\
R_{\delta}=\frac{\delta}{\mu_{0} S_{e}} .
\end{gathered}
$$




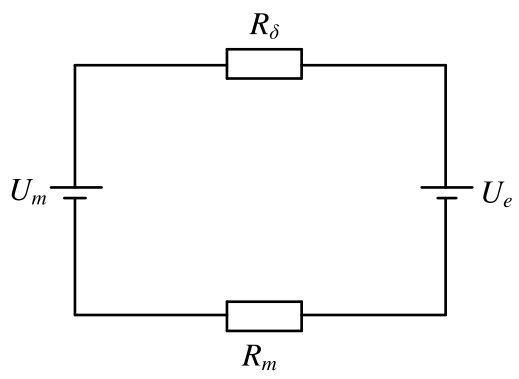

Fig. 4. Equivalent magnetic circuit model of the electromagnetic spring system.

The permanent magnet uses $\mathrm{NdFeB}$ material, which has a linear demagnetization curve. Hence, the magneto motive force and internal magnetoresistance can be taken as a constant:

$$
\begin{aligned}
& U_{m}=H_{c} L_{m}, \\
& R_{m}=\frac{H_{c} L_{m}}{B_{r} S_{m}} .
\end{aligned}
$$

Then, based on the ampere loop law in Maxwell's equations:

$$
\Phi=\frac{U}{R}
$$

The magnetic flux $\Phi$ is equivalent to the current in the circuit, the magneto motive force $U$ is equivalent to the electromotive force, and the magnetoresistance $R$ is equivalent to the resistance in the circuit. Obviously, equation (5) is similar to the Ohm's law in form, so this equation is defined as the Ohm's law of the magnetic circuit.

According to equation (5), it can be roughly assumed that the magnetic substance that generates magnetic field are connected along the specified direction, and the sum of the magneto motive forces is the algebraic sums of the magneto motive force of each magnetic substance. Therefore, the air gap magnetic flux of electromagnetic spring could be computed as:

$$
\Phi_{\delta}=\frac{U_{e}+U_{m}}{R_{m}+R_{\delta}} .
$$

Substituting equations (1)-(4) into (6):

$$
\Phi_{\delta}=\frac{I N+H_{c} L_{m}}{\frac{\delta}{\mu_{0} S_{e}}+\frac{H_{c} L_{m}}{B_{r} S_{m}}} .
$$

In order to facilitate the configuration and control of the vibration isolation system, let $S_{e}=S_{m}=S$, and the air gap magnetic flux of the electromagnetic spring is determined as:

$$
\Phi_{\delta}=\frac{\mu_{0} B_{r} S\left(I N+H_{c} L_{m}\right)}{B_{r} \delta+\mu_{0} H_{c} L_{m}} .
$$

On the basis of equation (8), the magnetic density of system is:

$$
B=\frac{\Phi_{\delta}}{S}=\frac{\mu_{0} B_{r}\left(I N+H_{c} L_{m}\right)}{B_{r} \delta+\mu_{0} H_{c} L_{m}} .
$$

Based on the design and calculation of the magnetic circuit, the equation of solving the magnitude of magnetic force is simplified as:

$$
F=\frac{B^{2} S}{2 \mu_{0}}
$$

In equation (10), magnetic induction intensity is $B$, also called the magnetic density, the effective area of magnetic pole is $S$, and the air magnetic permeability is $\mu_{0}$.

According to Coulomb's law, the repulsive force and attractive force are equal in absolute value. Therefore, as long as the magnetic density of the permanent magnet remains unchanged, the calculation of the attractive force can be converted into the repulsive force. And this feature is basically consistent with magnetic materials with a linear demagnetization curve.

By substituting equation (9) into equation (10), the electromagnetic force between the electromagnetic springs could be calculated as:

$$
F=\frac{\mu_{0} B_{r}^{2} S}{2}\left(\frac{I N+H_{c} L_{m}}{B_{r} \delta+\mu_{0} H_{c} L_{m}}\right)^{2} .
$$

According to equation (11), it is shown that the electromagnetic force is proportional to the square of the current and inversely proportional to the square of the air gap, which is also the nature of nonlinear of the electromagnetic spring system; and this nonlinear relationship increases the difficulty to design the control system.

Then assuming that the resistance of the electromagnet coil is $R$, the voltage loop equation at any time can be expressed as:

$$
u(t)=R I(t)+N \frac{d \Phi_{\delta}}{d t} .
$$

Also, when the system is in the quasi-equilibrium position, the straight leaf spring with no deformation, so the dynamic equation of the electromagnetic spring system in the vertical direction could be derived by the Newton's second law (taking the vertical upward for the positive direction of the system):

$$
m \frac{d^{2} \delta(t)}{d t^{2}}=F(\delta, I)-m g .
$$

At the quasi-equilibrium position $\left(\delta_{0}, I_{0}\right)$ :

$$
F\left(\delta_{0}, I_{0}\right)=m g .
$$

Finally, assuming that the electromagnetic spring system does not have vertical offset or dynamic deformation, the nonlinear mathematical model of the system in the vertical direction can be expressed by the following three 
equations:

$$
\left\{\begin{array}{l}
m \frac{d^{2} \delta(t)}{d t^{2}}=F(\delta, I)-m g \\
u(t)=R I(t)+N \frac{d \Phi_{\delta}}{d t} \\
F=\frac{\mu_{0} B_{r}^{2} S}{2}\left(\frac{I N+H_{c} L_{m}}{B_{r} \delta+\mu_{0} H_{c} L_{m}}\right)^{2}
\end{array} .\right.
$$

\subsection{Mathematical model based on linearization of equilibrium}

Since equation (15) is nonlinear, in order to utilize the developed linear control theory to analyze and design the electromagnetic springs control system, equation (15) is usually linearized by using the Taylor series expansion theorem near the equilibrium point $\left(\delta_{0}, I_{0}\right)$, and thus the approximate linear model of the system could be developed. Both theory and practice have proved that this local linearization has practical significance.

Equation (11) is linearized near the equilibrium point $\left(\delta_{0}, I_{0}\right)$ based on Taylor series expansion theorem, and ignored higher order infinitesimal terms above the second order as:

$$
F(\delta, I)=F\left(\delta_{0}, I_{0}\right)+k_{I} \Delta I(t)+k_{\delta} \Delta \delta(t) .
$$

In equation (16):

$$
\begin{gathered}
k_{I}=\left.\frac{\partial F(\delta, I)}{\partial I}\right|_{\left(\delta_{0}, I_{0}\right)}=\frac{\mu_{0} N B_{r}^{2} S\left(N I_{0}+H_{c} L_{m}\right)}{\left(B_{r} \delta_{0}+\mu_{0} H_{c} L_{m}\right)^{2}}, \\
k_{\delta}=\left.\frac{\partial F(\delta, I)}{\partial \delta}\right|_{\left(\delta_{0}, I_{0}\right)}=-\frac{\mu_{0} B_{r}^{3} S\left(N I_{0}+H_{c} L_{m}\right)^{2}}{\left(B_{r} \delta_{0}+\mu_{0} H_{c} L_{m}\right)^{3}}, \\
\Delta I(t)=I(t)-I_{0}, \\
\Delta \delta(t)=\delta(t)-\delta_{0} .
\end{gathered}
$$

$\Delta I(t)$ and $\Delta \delta(t)$ are the current and the air gap of the relative equilibrium position, respectively.

When the system is at an equilibrium position, substituting equation (8) into equation (12):

$$
\begin{aligned}
u(t)= & R I(t)+\left.N \frac{\partial \Phi_{\delta}}{\partial I(t)} \frac{d I(t)}{d t}\right|_{\left(\delta_{0}, I_{0}\right)}+\left.N \frac{\partial \Phi_{\delta}}{\partial \delta(t)} \frac{d \delta(t)}{d t}\right|_{\left(\delta_{0}, I_{0}\right)} \\
= & R I(t)+\frac{\mu_{0} N^{2} B_{r} S}{B_{r} \delta_{0}+\mu_{0} H_{c} L_{m}} \frac{d I(t)}{d t} \\
& -\frac{\mu_{0} N B_{r}^{2} S\left(I_{0} N+H_{c} L_{m}\right)}{\left(B_{r} \delta_{0}+\mu_{0} H_{c} L_{m}\right)^{2}} \frac{d \delta(t)}{d t}
\end{aligned}
$$

On the basis of electrotechnics theory, the inductance of the electromagnet coil can be determined as:

$$
L=N \frac{\partial \Phi_{\delta}}{\partial I(t)}=\frac{\mu_{0} N^{2} B_{r} S}{B_{r} \delta+\mu_{0} H_{c} L_{m}} .
$$

Thus, the inductance of the coil at the equilibrium position is defined as:

$$
L_{0}=\frac{\mu_{0} N^{2} B_{r} S}{B_{r} \delta_{0}+\mu_{0} H_{c} L_{m}} .
$$

By comparing equations (17), (21) and (23):

$$
u(t)=R I(t)+L_{0} \frac{d I(t)}{d t}-k_{I} \frac{d \delta(t)}{d t} .
$$

Substituting equations (19) and (20) into equations (13) and (24) as:

$$
\left\{\begin{array}{l}
m \frac{d^{2} \Delta \delta(t)}{d t^{2}}=F(\delta, I)-m g \\
u(t)=R\left[\Delta I(t)+I_{0}\right]+L_{0} \frac{d \Delta I(t)}{d t}-k_{I} \frac{d \Delta \delta(t)}{d t}
\end{array} .\right.
$$

Considering that the boundary conditions at the equilibrium point are:

$$
\left\{\begin{array}{l}
F\left(\delta_{0}, I_{0}\right)=m g \\
u_{0}=R I_{0}
\end{array} .\right.
$$

Substituting the boundary conditions into (16) and (25) results in:

$$
\left\{\begin{array}{l}
m \frac{d^{2} \Delta \delta(t)}{d t^{2}}=k_{I} \Delta I(t)+k_{\delta} \Delta \delta(t) \\
\Delta u(t)=R \Delta I(t)+L_{0} \frac{d \Delta I(t)}{d t}-k_{I} \frac{d \Delta \delta(t)}{d t}
\end{array}\right.
$$

where $\Delta u=u(t)-u_{0}$ is the voltage of the relatively equilibrium position. Thus, equation (27) is the linearization equation based on equilibrium point of equation (15), i.e. the linearized mathematical model of the system.

\section{The design of electromagnetic spring control system}

If the current is the control input variable, letting $(\Delta \delta, \Delta \dot{\delta})$ as the state variable, and $\Delta \delta_{0}=0, \Delta \dot{\delta}_{0}=0$ at the equilibrium position, based on equation (27), the state-space representation of the system could be written as:

$$
\left[\begin{array}{c}
\Delta \dot{\delta} \\
\Delta \ddot{\delta}
\end{array}\right]=\left[\begin{array}{cc}
0 & 1 \\
\frac{k_{\delta}}{m} & 0
\end{array}\right] \cdot\left[\begin{array}{c}
\Delta \delta \\
\Delta \dot{\delta}
\end{array}\right]+\left[\begin{array}{c}
0 \\
\frac{k_{I}}{m}
\end{array}\right] \cdot \Delta I .
$$




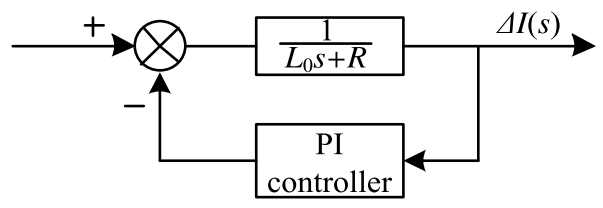

Fig. 5. Structure diagram of current loop.

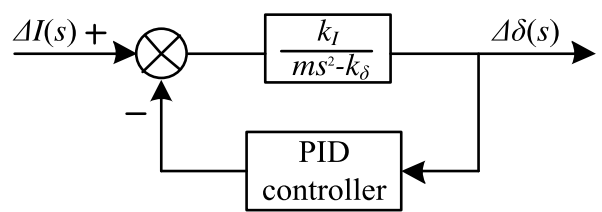

Fig. 6. Structure diagram of position loop.

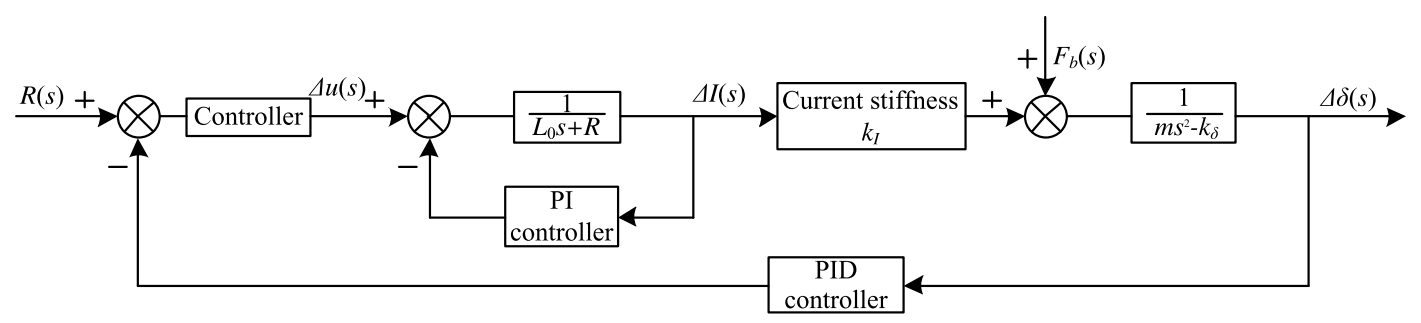

Fig. 7. Structure diagram of control system with double closed-loop.

By operating equation (28) on the basis of the Laplace transform, the open-loop transfer function of the system is:

$$
G_{I}(s)=\frac{k_{I}}{m s^{2}-k_{\delta}} .
$$

According to the characteristic equation of the system and the Routh criterion, it is a second-order unstable system. It is necessary to add a feedback loop to achieve the stable control for the electromagnetic spring. However, the control method of using current as a control input variable reduces the system from third order to second-order, which is relatively convenient and easy to implement when designing a controller.

In conclusion, the control method of using current control input variable is applied in this paper. However, in practical situation, the electromagnetic is still driven by the chopper. The chopper with high current response speed is the precondition of reducing the complexity of system control by the control method of using current control input variable. Therefore, to improve the current response speed, the current loop should be added into the chopper. And the current response speed improves by configuring current loop appropriately. According to this requirement, the double closed-loop feedback control system with position feedback outer loop and current feedback inner loop is employed to control the electromagnetic spring steadily in this paper.

The transfer function of the traditional PID controller is expressed as:

$$
G_{j}(s)=K_{P}+\frac{K_{I}}{s}+K_{D} s .
$$

In this equation, $K_{P}$ is called as the proportional gain, $K_{I}$ is named as the integral gain, and $K_{D}$ is entitled as the differential gain. In PID controller, the three parameters are alternative, and thus different combinations of controllers can be obtained under different values.
In terms of the design of the inner current loop, using PI controller, its structure diagram is shown in Figure 5.

And the closed-loop characteristic equation of the controller can be written as:

$$
\frac{L_{0}}{K_{I}} s^{2}+\frac{K_{P}+R}{K_{I}} s+1=0 .
$$

Obviously, the equation (31) expresses a typical secondorder system, which could realize the good dynamic and static performance of the current loop by appropriately choosing the parameter $K_{P}$ and $K_{I}$.

In terms of the design of the outer position loop, based on equation (29), using PID controller, its structure diagram is shown in Figure 6.

And the closed-loop characteristic equation of the controller is expressed as

$$
s^{3}+\frac{k_{I} K_{D}}{m} s^{2}+\frac{k_{I} K_{P}-k_{\delta}}{m} s+\frac{k_{I} K_{I}}{m}=0 .
$$

Notably, equation (32) expresses a typical third-order system, which could realize the good control performance of the position by appropriately choosing the parameter $K_{P}, K_{I}$ and $K_{D}$.

The target of the vibration isolation controller is to isolate the objects from the external vibration disturbance, i.e. makes effort to keep the objects at the equilibrium position. After adding external disturbance, the structure diagram of control system is shown in Figure 7.

\section{The dynamic and stiffness analysis of electromagnetic spring system}

After adding disturbance $y_{b}$, the dynamic equation of electromagnetic spring system is:

$$
m \frac{d^{2} \Delta \delta(t)}{d t^{2}}=k_{I} \Delta I(t)+k_{\delta}\left[\Delta \delta(t)+y_{b}(t)\right]
$$


Let $F(t)=k_{\delta} y_{b}(t)$, by operating equation (33) on the basis of the Laplace transform:

$$
\frac{\Delta \delta(s)}{F(s)}=\frac{1}{m s^{2}-k_{\delta}-k_{I} G_{j}(s)} .
$$

Substituting equation (30) into equation (34):

$$
\frac{\Delta \delta(s)}{F(s)}=\frac{1}{m s^{2}-k_{\delta}-k_{I} K_{P}-\frac{k_{I} K_{I}}{s}-k_{I} K_{D} s} .
$$

Let $s=i \omega$. The frequency transfer function of system is:

$$
T_{\delta}(i \omega)=\frac{1}{\left(-k_{\delta}-k_{I} K_{P}-m \omega^{2}\right)+i \omega\left(\frac{k_{I} K_{I}}{\omega^{2}}-k_{I} K_{D}\right)} .
$$

For spring-mass-damper system, the general frequency transfer function is:

$$
T(i \omega)=\frac{1}{k-m \omega^{2}+i \omega c} .
$$

By comparing equations (36) and (37), the equivalent stiffness and equivalent damping coefficient of electromagnetic spring system can be expressed as:

$$
\left\{\begin{array}{l}
k_{e}=-k_{\delta}-k_{I} K_{P} \\
c_{e}=\frac{k_{I} K_{I}}{\omega^{2}}-k_{I} K_{D}
\end{array} .\right.
$$
is:

The damping ration of electromagnetic spring system

$$
\zeta=\frac{c_{e}}{2 \sqrt{m k_{e}}}=\frac{\frac{k_{I} K_{I}}{\omega^{2}}-k_{I} K_{D}}{2 \sqrt{m\left(-k_{\delta}-k_{I} K_{P}\right)}} .
$$

As shown in equation (38), the equivalent stiffness and equivalent damping are not only relevant to the structural parameter $k_{I}$ and $k_{\delta}$, but also closely related to parameter $K_{P}, K_{I}$ and $K_{D}$ of the controller. In addition, the equivalent damping is a function of the system frequency, and the equivalent damping increases as frequency decreases. Therefore, by adjusting the parameter of control system to change the stiffness and damping of vibration isolation system, which can achieve more effective vibration isolation.

This paper achieves effective vibration isolation by changing the equivalent stiffness of system. Substituting equations (17) and (18) into the first equation of equation (38), the equivalent stiffness could be expressed as:

$$
k_{e}=\frac{\mu_{0} B_{r}^{3} S\left(N I+H_{c} L_{m}\right)^{2}}{\left(B_{r} \delta+\mu_{0} H_{c} L_{m}\right)^{3}}-\frac{\mu_{0} N B_{r}^{2} S\left(N I+H_{c} L_{m}\right)}{\left(B_{r} \delta+\mu_{0} H_{c} L_{m}\right)^{2}} K_{P} .
$$

As shown in equation (40), the equivalent stiffness is only in connection with control parameter $K_{P}$, current $I$ and air gap $\delta$ when the specifications, materials of the permanent magnet and the size of the electromagnet, the

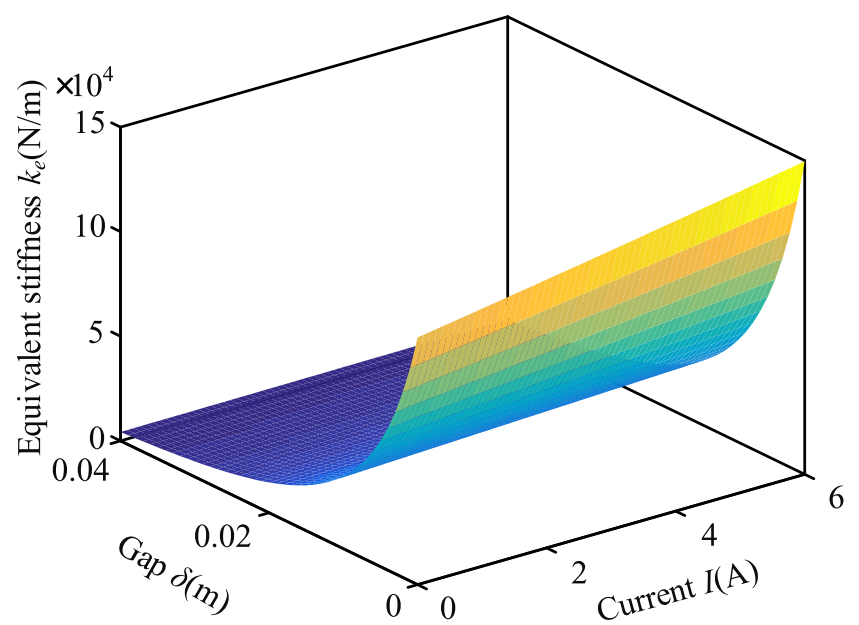

Fig. 8. Cloud diagram of equivalent stiffness under different currents and air gaps.

number of coil turns are selected. The control parameter $K_{P}$ has been determined when debugging control system, and thus the equivalent stiffness varies with the variation of current $I$ and air gap $\delta$ when the system operating. The cloud diagram of equivalent stiffness at different currents and air gaps is shown in Figure 8.

As illustrated in Figure 8, the equivalent stiffness of the system increases as the air gap decreases and the current increases, and decreases as the air gap increases and the current decreases.

Considering the mass-varying objects to maintain the straight leaf spring with no deformation at the equilibrium position, i.e. the air gap between electromagnetic springs remains constant at the equilibrium, which requires controlling the electromagnetic force by changing the current, and also the changes of current would lead to the changes of system stiffness. At the equilibrium position, putting equation (14) into equation (40), regarding the current $I_{0}$ as the function of mass $m$, the equivalent stiffness of system for mass-varying objects can be expressed as:

$$
k_{e}=\frac{2 m g B_{r}-N B_{r} K_{P} \sqrt{2 m g \mu_{0} S}}{B_{r} \delta_{0}+\mu_{0} H_{c} L_{m}} .
$$

In Figure 9, when the mass of objects changes, the equivalent stiffness of system also alters, and they are in linear relationship approximately, i.e. the natural frequency of system nearly remains unchanged. Therefore, it can be concluded that for mass-varying objects, the vibration isolation system can still exert good vibration isolation performance in the low frequency band.

\section{Simulation and analysis of the performance of vibration isolation system}

\subsection{Time responses of sinusoidal wave excitation}

The vibration isolation control system is built on the basis of the system control structure diagram shown in Figure 7 by using Simulink software. The performance of current 


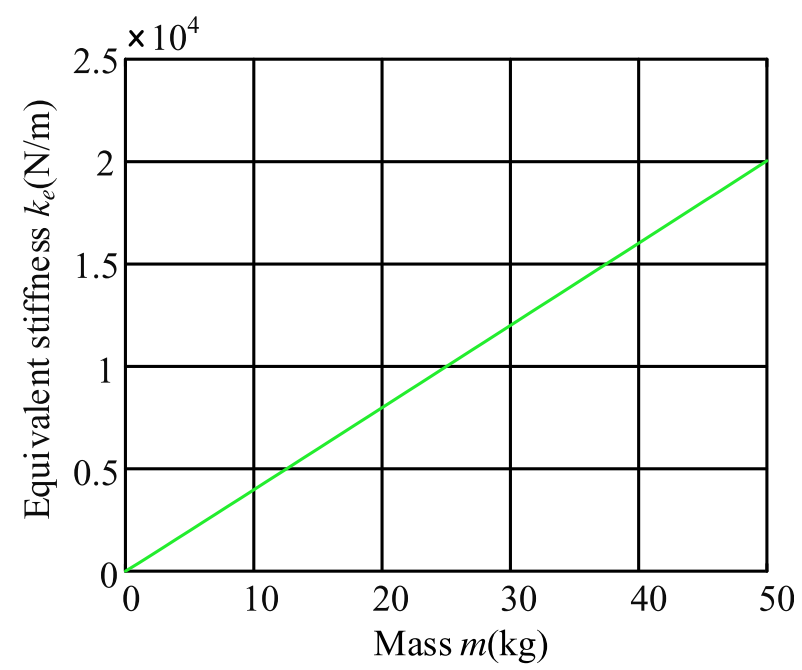

Fig. 9. Equivalent stiffness curves of different mass.

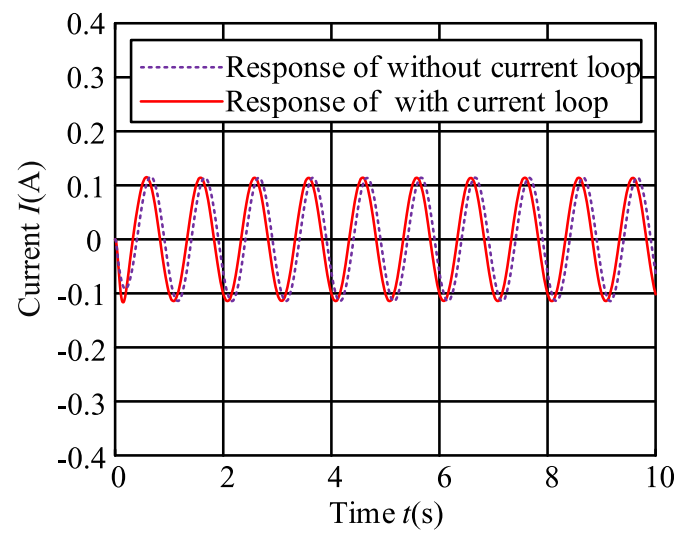

Fig. 10. Current curve under sinusoidal excitation.

control directly affects the vibration isolation effect of semi-active vibration isolator because the change of electromagnetic force is achieved by controlling the current in the electromagnetic coil. Firstly, a sinusoidal excitation with amplitude of $3 \mathrm{~mm}$ and a frequency of $1 \mathrm{~Hz}$ is, respectively, applied to the control system with inner current loop and the control system without inner current loop, then the response curve of current in the coil could be derived as shown in Figure 10. Comparing the current response in the figure, it is concluded that the current response without current loop control is slightly delayed compared to the current response controlled by current loop (Tab. 1).

Then the amplitude response curve of the semi-active vibration isolation system with current loop control and without current loop control can be obtained, as shown in Figure 11a. The blue curve is the input sine excitation, the green curve is the system response curve without current loop control, and the red curve is the system response curve with current loop control. By comparison, the system response without current loop control has a hysteresis about half a period, while the amplitude of the system with
Table 1. Basic parameters of semi-active vibration isolation system.

\begin{tabular}{|c|c|c|c|}
\hline Name & Symbol & Value & Unit \\
\hline Air permeability & $\mu_{0}$ & $4 \pi \times 10^{-7}$ & $\overline{\mathrm{Nm} / \mathrm{A}^{2}}$ \\
\hline $\begin{array}{l}\text { Magnetic induction } \\
\text { strength }\end{array}$ & $B_{r}$ & 1.21 & $\mathrm{~T}$ \\
\hline Coercive force & $H_{c}$ & 960 & $\mathrm{kA} / \mathrm{m}$ \\
\hline $\begin{array}{l}\text { Effective cross-sectional } \\
\text { area }\end{array}$ & $S$ & 0.002 & $\mathrm{~m}^{2}$ \\
\hline $\begin{array}{l}\text { Effective length of the } \\
\text { permanent magnet }\end{array}$ & $L_{m}$ & 0.02 & $\mathrm{~m}$ \\
\hline Gravity acceleration & $g$ & 10 & $\mathrm{~m} / \mathrm{s}^{2}$ \\
\hline
\end{tabular}

current loop control is greatly reduced, and the response delay is very small. Hence, the introduction of current loop improves the vibration isolation performance of the semiactive vibration isolation system, and reduces the response delay and the displacement transmissibility.

Subsequently, the multi-frequency sine excitation is exerted on the system, the response curve of the system with current loop control and without current loop control is shown in Figure 11b. It is displayed that the system response with current loop control is more stable under the effect of multi-frequency sine excitation, which manifests that the introduction of current loop to the semi-active vibration isolation system increases the stability of the system response.

\subsection{Time responses of unit pulse excitation}

Similarly, a unit pulse excitation is applied to a control system with current loop and a control system without current loop, two amplitude response curves of system are shown in Figure 12. Noticeably, the amplitude response peak of semi-active vibration isolation system without current loop is approximately $0.14 \mathrm{~mm}$, the adjustment time is about $1 \mathrm{~s}$. However, the amplitude response peak with current loop is approximately $0.04 \mathrm{~mm}$ and the adjustment time is about $0.5 \mathrm{~s}$. Consequently, the semiactive vibration isolation system has good control performance, and the introduction of current loop could effectively shorten the adjustment time and further reduce the amplitude response.

\subsection{Amplitude-frequency responses of sinusoidal wave excitation}

According to the frequency transfer function of system shown in equation (36), the amplitude-frequency characteristic of the system can be expressed as:

$$
A(\omega)=\frac{1}{\sqrt{\left(-k_{\delta}-k_{I} K_{P}-m \omega^{2}\right)^{2}+\left[\omega\left(\frac{k_{I} K_{I}}{\omega^{2}}-k_{I} K_{D}\right)\right]^{2}}} .
$$




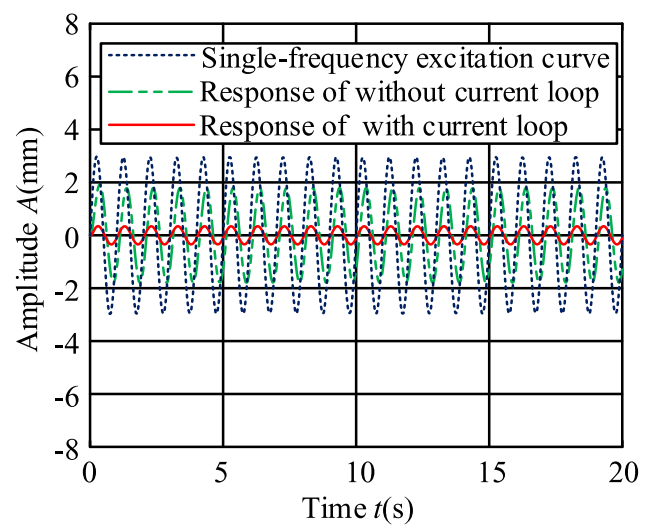

(a)

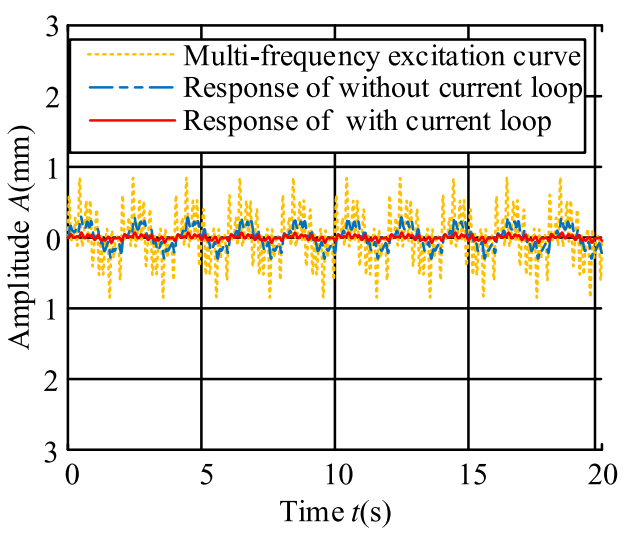

(b)

Fig. 11. Response curves of semi-active vibration isolation systems. (a) Single-frequency sinusoidal excitation input; (b) multifrequency sinusoidal excitation input.

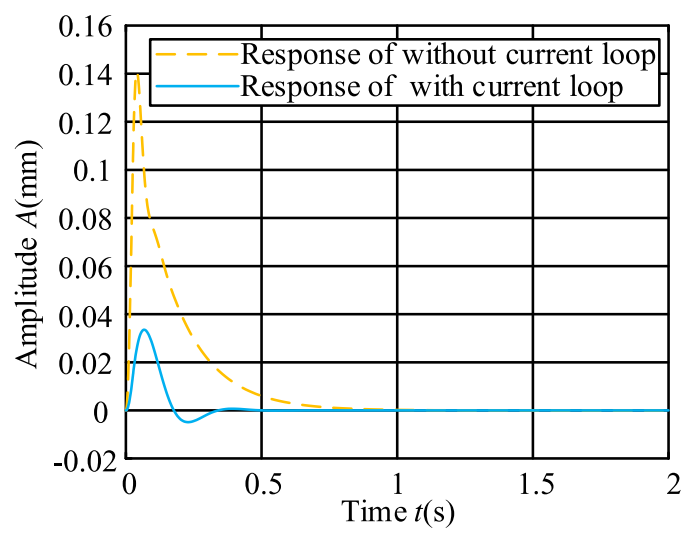

Fig. 12. Unit pulse excitation response curve of semi-active vibration isolation system.

On the basis of equation (42), the amplitude-frequency characteristics of the semi-active vibration isolation system with variable-parameter and the general vibration isolation system with fixed-parameter for mass-varying objects are drawn respectively, as shown in Figure 13.

It can be noticed from the figure about the general vibration isolation system with fixed-parameter, when the mass of objects increases, the resonance frequency shifts to the left, and the peak value increases, which shows that the general vibration isolation system with fixed-parameters has certain limitations of the vibration isolation for massvarying objects, i.e. with the increase of the mass, the vibration isolation performance for low frequency interference is reduced. On the contrary, the semi-active vibration isolation system with variable-parameter could solve the problem effectively. When the mass of object changes, the resonance frequency remains almost unchanged, and the peak value decreases with the increase of the mass, and it has a good vibration isolation effect in the low frequency band from 0 to $10 \mathrm{~Hz}$. It proves that the semi-active vibration isolation system can maintain the good vibration isolation effect on the low-frequency band or the specificfrequency band for mass-varying objects, and meets our requirements.

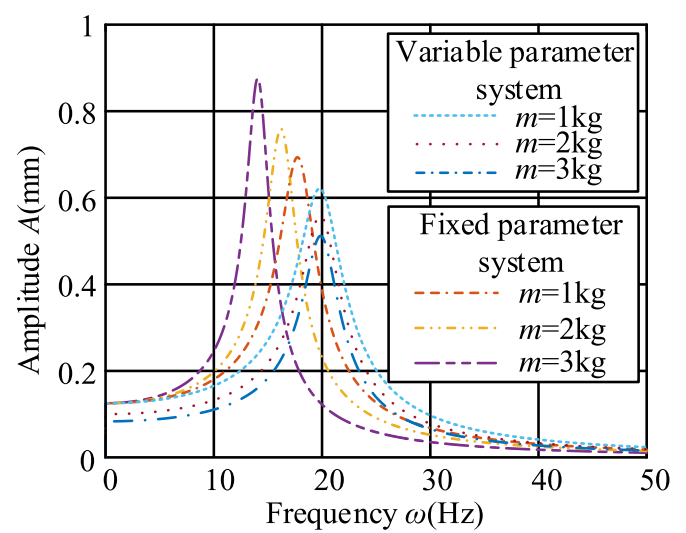

Fig. 13. Amplitude-frequency characteristic curve of semiactive vibration isolation system.

As shown in Figure 14, the effect of the control parameters on the amplitude-frequency characteristics is shown. In Figure 14a, the peak value decreases as the $K_{D}$ increases; in Figure 14b, as the $K_{P}$ increases, the peak corresponding to the frequency is reduced, i.e. the resonant frequency is reduced, which provides an effective adjustment method for optimizing the vibration isolation frequency band and performance of the semi-active vibration isolation system.

\section{Experimental investigation}

According to the above theoretical research, the experimental verification of the proposed semi-active vibration isolation system is now carried out. The excitation control device of the vibration isolation experiment is shown in Figure 15, and Figure 16 shows the schematic diagram of the vibration isolation experiment process.

The displacement transmissibility was selected as the parameter for studying the vibration isolation performance for vibration isolation experiments. Firstly, the vibrationisolating object is selected to be $1 \mathrm{~kg}$, and the excitation 


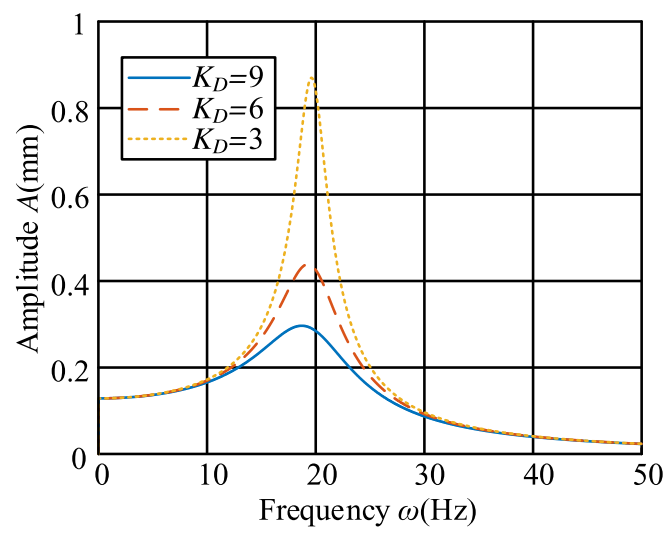

(a)

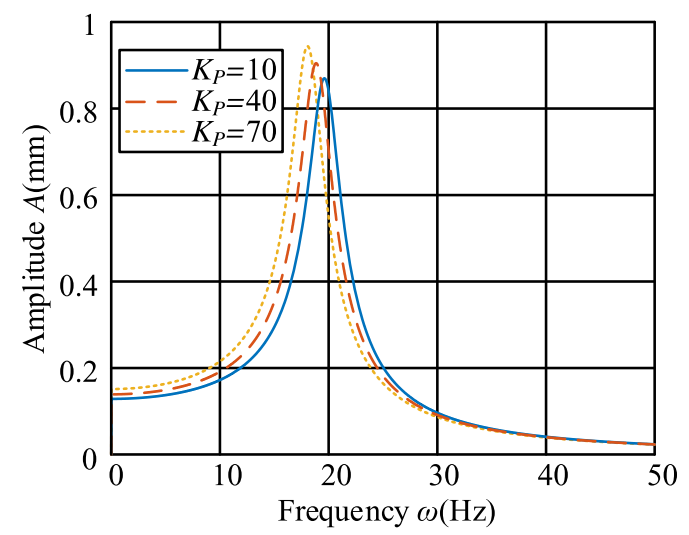

(b)

Fig. 14. Effect of control parameters on amplitude-frequency characteristics.

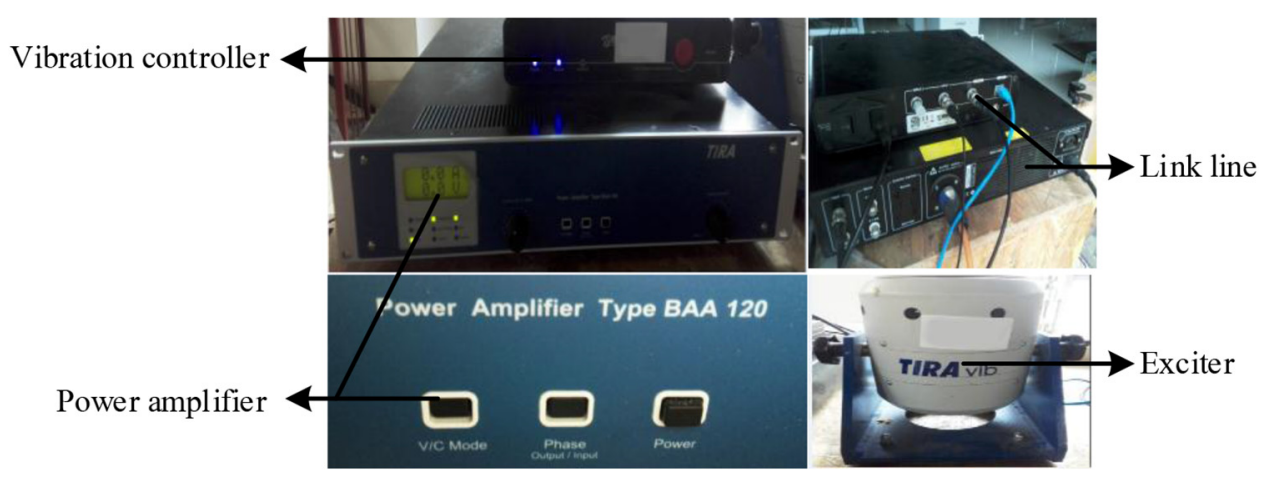

Fig. 15. Excitation control device of the vibration isolation experiment.

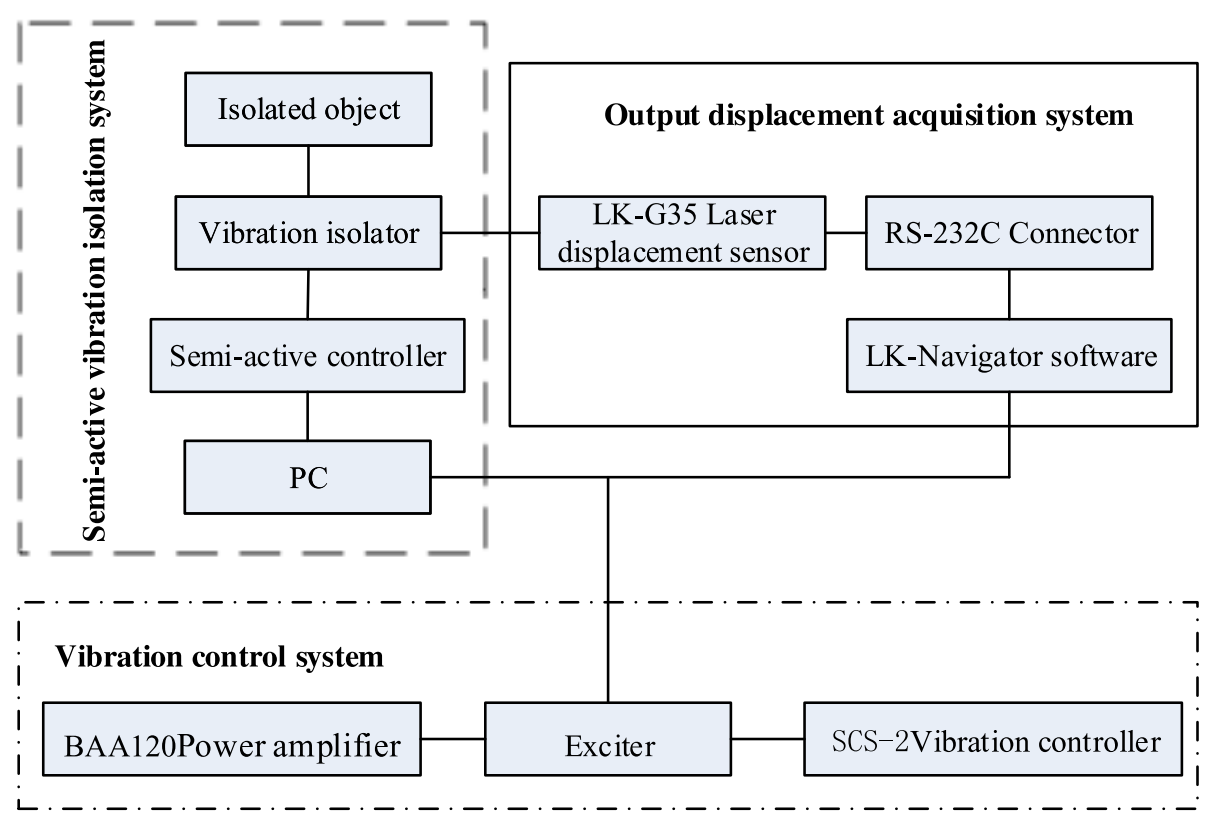

Fig. 16. Schematic diagram of the vibration isolation experiment process. 


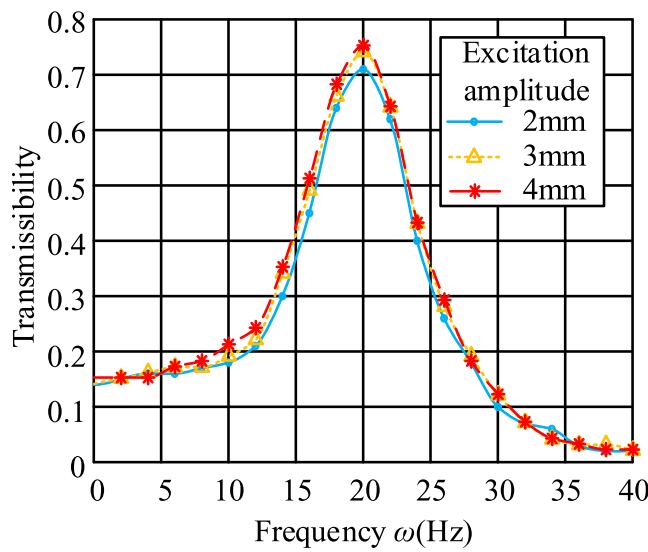

(a)

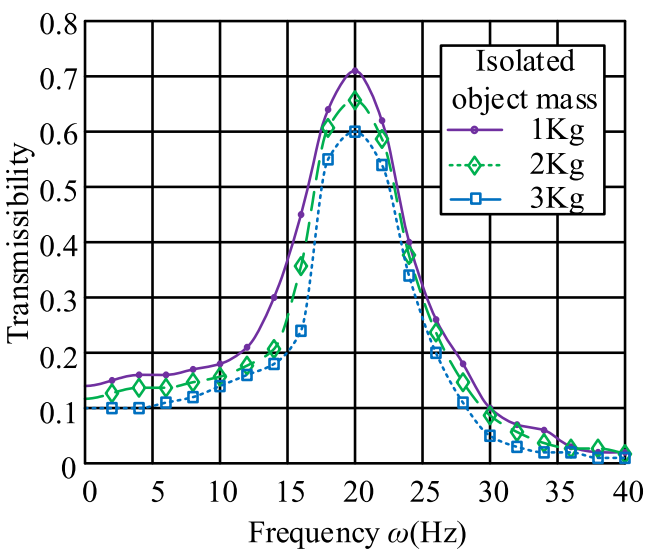

(b)

Fig. 17. Displacement transmissibility curve. (a) Transmissibility curve for different excitation amplitudes; (b) transmissibility curve for different vibration-isolated objects.

amplitudes are $2 \mathrm{~mm}, 3 \mathrm{~mm}$ and $4 \mathrm{~mm}$, respectively. Then, the selected excitation amplitude is $2 \mathrm{~mm}$, and the vibration-isolating objects are $1 \mathrm{~kg}, 2 \mathrm{~kg}$, and $3 \mathrm{~kg}$, respectively. The corresponding displacement transmissibility curve is shown in Figure 17.

As shown in Figure 17a, when the excitation frequency increases gradually, the displacement transmissibility curve of the semi-active vibration isolation system first increases and then decreases, finally stabilizes, which is consistent with the theoretical analysis; at the same time, as the magnitude of the excitation increases, the peak of the displacement transmissibility also increases. The displacement transmissibility of the isolated objects with different masses is shown in Figure 17b. It shows when the mass of object changes, the resonance frequency remains almost unchanged, and the peak value decreases with the increase of the mass. It also proves the correctness of the theoretical analysis shown in Figure 13, and it has a good vibration isolation effect in the low frequency band from 0 to $10 \mathrm{~Hz}$.

\section{Conclusion}

According to the results of simulation analysis, under the effect of single-frequency and multi-frequency sinusoidal excitation, the speed and stability of current response has been improved, the response delay of vibration isolation system has been decreased, and the stability of system response has been distinctly improved. Likewise, under the unit pulse excitation, the system of using current loop could reduce the adjustment time by approximately half compared to the system without current loop. It can clearly be seen that the current loop control could further improve the performance of vibration isolation system. Finally, the amplitude-frequency performance of the semi-active vibration isolation system is analyzed and the experiments prove the correctness of the theory. It is shown that for the general vibration isolation system with fixed-parameter, the natural frequency descends with mass increase, which weakens the vibration isolation at low frequency band. By contrast, the natural frequency of the semi-active vibration isolation system with variableparameter is not affected by the mass change and it effectively suppresses low-frequency vibration from 0 to $10 \mathrm{~Hz}$ for mass-varying objects, so it reflects the superiority of semi-active vibration isolation system.

Acknowledgments. This work was supported by the National Natural Science Foundation of China (Grant No.51305444).

\section{References}

[1] T.D. Le, M.T.N. Bui, K.K. Ahn, Improvement of vibration isolation performance of isolation system using negative stiffness structure, IEEE. Trans. Mechatron. 21, 1561-1571 (2016)

[2] T.D. Le, K.K. Ahn, A vibration isolation system in low frequency excitation region using negative stiffness structure for vehicle seat, J. Sound Vib. 330, 6311-6335 (2011)

[3] X.-X. Bai et al., Integrated semi-active seat suspension for both longitudinal and vertical vibration isolation, J. Intell. Mater. Syst. Struct. 28, 1036-1049 (2017)

[4] B. Kasemi et al., Fuzzy-PID controller for semi-active vibration control using magnetorheological fluid damper, Procedia Eng. 41, 1221-1227 (2012)

[5] B. Yang, Y. Hu, H. Fang, Research on arrangement scheme of magnetic suspension isolator for multi-degree freedom vibration isolation system, J. Ind. Inf. Integr. 6, 47-55 (2017)

[6] S. Behrens, A.J. Fleming, S.O.R. Moheimani, Control orientated synthesis of electromagnetic shunt impedances for vibration isolation, IFAC Proc. 37, 301-306 (2004)

[7] T. Sun, Z. Huang, D. Chen, Signal frequency-based semiactive fuzzy control for two-stage vibration isolation system, J. Sound Vib. 280, 965-981 (2005)

[8] C. Min, M. Dahlmann, T. Sattel, A concept for semi-active vibration control with a serial-stiffness-switch system, J. Sound Vib 405, 234-250 (2017)

[9] A.M. Bazinenkov, V.P. Mikhailov, Active and semi active vibration isolation systems based on magnetorheological materials, Procedia Eng. 106, 170-174 (2015)

[10] Q. Meng, et al., Research and analysis of quasi-zero-stiffness isolator with geometric nonlinear damping, Shock Vib. 2017, 1-9 (2017) 
[11] D. Karnopp, Vibration control using semi-active force generators, J. Eng. Ind. 96, 619-626 (1974)

[12] E. Guglielmino, K.A. Edge, A controlled friction damper for vehicle applications, Control Eng. Pract. 12, 431-443 (2004)

[13] Y. Suda, T. Shiiba, K. Hio, Study on electromagnetic damper for automobiles with nonlinear damping force characteristics, Veh. Syst. Dyn. Suppl. 41, 637-646 (2004)

[14] Y. Liu, H. Matsuhisa, H. Utsuno. Semi-active vibration isolation system with variable stiffness and damping control, J. Sound Vib. 313, 16-28 (2008)

[15] T.-H. Wu, C.-C. Lan, A wide-range variable stiffness mechanism for semi-active vibration systems, J. Sound Vib. 363, 18-32 (2016)

[16] W. Wei, Design and simulation analysis of magnetorheological elastomers with variable stiffness isolation system, Manuf. Mech. 12, 22-25 ( 2011)

[17] V. Wickramasinghe, C. Yong, D. Zimeik, Smart spring: a novel adaptive impedance control approach for active vibration suppression applications, Proc. SPIE, 5390, 359-369 (2004)
[18] Z. Mei-ling, Y. Shi-feng, Y. Jing-ping, Theoretical study on electromagnetic spring in active vibration control, J. Southeast Univ. 11, 97-101 (1993)

[19] Z. Hong-tian, Z. Tian-yuan, L. Zhi-gang. Electromagnetic active vibration absorber and its characteristic experimental analysis, Sound Vib. Control 3, 37-38 (1995)

[20] R.-F. Fung, Y.-T. Liu, Dynamic model of an electromagnetic actuator for vibration control of a cantilever beam with a tip mass, J. Sound Vib. 288, 957-980 (2005)

[21] Z. Zhuo-liang, Research on design of adjustable vibration isolators, Mod. Vib. Noise Technol. 4, 137-141 (2005)

[22] T. Pranoto, K. Nagaya, Vibration suppression device using permanent-electromagnet and MRF shear damper, J. Mater. Process. Technol. 181, 235-240 (2007)

[23] W. Guang-wei, M. Lv-zhong, Z. Wei, Study on a new hybrid active magnetic vibration isolation system, J. Mach. Des. 25, 49-51 (2008)

[24] D. Easu, A. Siddharthan, Theoretical and experimental analysis of a vibration isolation system using hybrid magnet, Procedia Eng. 64, 1139-1146 (2013)

Cite this article as: J. Xu, X. Yang, W. Li, J. Zheng, Y. Wang, M. Fan, Research on semi-active vibration isolation system based on electromagnetic spring, Mechanics \& Industry 21, 101 (2020) 